\title{
COMPORTAMIENTO EPIDEMIOLOGICO DEL CÁNCER DE COLON EN EL ÁREA METROPOLITANA DE CÚCUTA 2001-2002
}

Por:

Alix Z Bohórquez O.' , Esperanza Gómez J.2, María Mercedes Ramírez O. ${ }^{3}$, Gloria Esperanza Zambrano P. ${ }^{3}$, Manuel Eduardo Moros ${ }^{4}$

\section{RESUMEN}

Con el propósito de identificar las características epidemiológicas y los factores de riesgo prevalentes para cáncer de colon en la población adulta de Cúcuta, se desarrollo el presente estudio de tipo descriptivo, prospectivo de corte transversal. La población de estudio estuvo constituida por 37 pacientes con este diagnóstico que asistieron a las instituciones de salud públicas y privadas del municipio de Cúcuta (N.S.), en el período 2001 - 2002. Se encontró que el riesgo se incrementa con la edad, el sexo más afectado es el femenino, el antecedente familiar de cáncer de colon, cáncer en otros órganos, poliposis y colitis ulcerativa se presentó en 5.4 de cada 10 pacientes del estudio. Se identificaron como factores de riesgo alimentarios el alto consumo de carne de res, ingesta de alimentos embutidos, la reutilización de los aceites en la cocción de los alimentos y el consumo de comidas recalentadas. Es ampliamente utilizada en la dieta la harina refinada de maíz para la elaboración y consumo de arepas. Los factores de riesgo en el estilo de vida que se presentaron con mayor porcentaje fueron el sedentarismo y el tabaquismo, seguido del consumo de alcohol y la obesidad.

Un alto porcentaje manifiesta haber tenido estreñimiento, diarrea o ambos síntomas. El 50\% refiere automedicación para el manejo de estos síntomas, empleando tanto medicamentos farmacológicos como el uso de la medicina casera. Como condiciones precursoras se encuentran el sangrado rectal con $73 \%$, la pérdida de peso con $63.6 \%$, seguido por la hemorroides y la anemia con $36.4 \%$. Se encontraron largos períodos de evolución de la enfermedad antes de que se definiera el diagnóstico.

Palabras Claves: Cáncer de colon, Factores de riesgo

\section{INTRODUCCIÓN}

El cáncer de colon y recto, también llamado cáncer colorectal, es el tercero más mortal entre todos los cánceres conocidos y supone 10\% de los detectados en hombres y un $11 \%$ de los que sufren las mujeres, según datos de la Sociedad Estadounidense del Cáncer.[1]

En el Norte de Santander los tumores malignos presentan una tasa de mortalidad en 1998 de 54.3 por 100.000 habitantes, ocupando el cáncer de colon el quinto lugar con tasa de mortalidad de $2.19 \mathrm{X}$ 100.000 habitantes, después de los tumores malignos de estómago, de cuello uterino, de bronquios o pulmón, de próstata y de mama. Para el año 2000 se observó un incremento en la tasa de mortalidad, ubicándose en la segunda causa de mortalidad por esta causa en el Norte de Santander.[2]

El Grupo de investigación en Salud Pública, interesado en el conocimiento de la problemática de Salud Pública regional desarrolla el presente proyecto con el objetivo de identificar las características epidemiológicas del evento en pacientes diagnosticados con cáncer de colon en el período 2001-2002 en el Área Metropolitana de Cúcuta.

\footnotetext{
'Enfermera Mg. Salud Pública. Directora GISP

${ }^{2}$ Enfermera Mg. Docente U.F.P.S.

${ }^{3}$ Enfermera Mg. Enfermería. Docente U.F.P.S.

${ }^{4}$ Médico, Especialista en Cirugía General. Centro de Rehabilitación Norte de Santander
} 
Comportamiento epidemiológico del cáncer de colon en el Área Metropolitana de Cúcuta 2001-2002

Alix Z Bohórquez O., Esperanza Gómez J., María Mercedes Ramírez O., Gloria Esperanza Zambrano P., Manuel Eduardo Moros

\section{MATERIALES Y METODOS}

El presente es un estudio de tipo descriptivo, prospectivo de corte transversal que busca identificar las características epidemiológicas del cáncer de colon en la población diagnosticada en el área metropolitana de Cúcuta en el período señalado. La población está constituida por 37 pacientes diagnosticados con cáncer de colon en las instituciones hospitalarias públicas y privadas de la ciudad de Cúcuta, en el período 2001 2002.

Como fuentes de información se utilizaron la historia clínicas y entrevistas estructurada a los pacientes.

Una vez obtenida la información de las historias clínicas: datos de identificación, historia familiar, revisión de reportes de patología, de endoscopia, descripción del acto quirúrgico, evolución del caso, características del tumor, y medidas terapéuticas adoptadas en cada caso, se consignaron en el instrumento diseñado para tal fin previamente validado mediante prueba piloto.

En la entravista se utilizó un cuestionario en el que se indagó sobre hábitos alimentarios, estilo de vida, signos y sintomas prévios al diagnóstico, manejo y pesquisa que se realizó. Mediante una pregunta abierta se consignaron las recomendaciones de los pacientes relacionadas con el mejoramiento de la atención.

En razón a que 15 pacientes ya habían fallecido o no fue posible su ubicación, la información complementaria recolectada mediante entrevista estructurada, se realizó a 22 pacientes ubicados en sus domicilios o en los centros asistenciales.

\section{RESULTADOS}

De acuerdo a los resultados obtenidos se observó un incremento de los casos a partir de los 50 años, en una razón de 3: 1 por cada menor de 50 años, respecto del sexo de los pacientes se encontró que el sexo femenino predomina con 20 de los 37 casos del estudio, con una relación hombre mujer de 1:1,2
Aunque los factores medioambientales son importantes en el desarrollo de cáncer colorectal, el riesgo también puede heredarse. La historia familiar es un factor de riesgo importante: los individuos con un solo pariente del primer grado con cáncer colorectal tienen un riesgo doble de desarrollar este cáncer, incrementándose el riesgo cuando existe el antecedente de más parientes afectados [1,3]. El presente estudio reportó el antecedente familiar de cáncer de colon en 1 de cada 12 pacientes. Los factores de riesgo para cáncer de colon: poliposis y colitis ulcerativa se presentaron en dos de los casos. En general los antecedentes familiares de patologías de riesgo para esta entidad se presentaron en 5.4 de cada 10 pacientes del estudio.

Al analizar los aspectos relacionados con la alimentación se encontró que el $77 \%$ de la población del estudio utilizan gas como combustible para la cocción de los alimentos considerándose este como un factor protector. De otra parte, la reutilización del aceite en la dieta de 8 de cada 10 pacientes, Tabla 1, se constituye en un dato significativo, considerando que esta práctica ha sido identificada como de riesgo para cáncer de colon al acelerar la modificación de los químicos presentes en las grasas que generan mutaciones cancerígenas. La grasa más empleada para la preparación de los alimentos es el aceite vegetal y aunque esta es considerada de menor riesgo que las de origen animal, los estudios han reportado que en general la dieta rica en grasa es factor de riesgo para cáncer de colon.

Tabla 1. Reutilización de aceites en la cocción

\begin{tabular}{|c|c|c|}
\hline $\begin{array}{c}\text { USO DE ACEITE } \\
\text { REUTILIZADO }\end{array}$ & $\mathrm{N}$ & $\%$ \\
\hline $\mathrm{SI}$ & 18 & 82 \\
\hline $\mathrm{NO}$ & 4 & 18 \\
\hline TOTAL & 22 & 100 \\
\hline
\end{tabular}

Fuente: Entrevista

Revista Respuestas - Universidad Francisco de Paula Santander Año 9 No. 2 
Comportamiento epidemiológico del cáncer de colon en el Área Metropolitana de Cúcuta 2001-2002

Alix Z Bohórquez O., Esperanza Gómez J., María Mercedes Ramírez O.,, Gloria Esperanza Zambrano P., Manuel Eduardo Moros

Teniendo en cuenta que los factores dietéticos parecen ser los determinantes más importantes de riesgo de cáncer colorectal $[4,5]$ y de acuerdo a la información recolectada en el presente estudio, se establece que el mayor consumo de carne fue la de res en 8 de cada 10 pacientes, seguido el consumo de pollo con 4 de cada 10, coincidiendo con diversos estudios que han encontrado que la dieta con alto contenido en carnes y grasas animales parece incrementar el riesgo de cáncer de colon, especialmente las carnes rojas como lo afirman Giovannucci E., Rimm Eb y colaboradores en su obra "Consumo de grasa, carne y fibra en relación con el riesgo de cáncer colorectal en el hombre"[6], ver gráfico 1. Cabe resaltar los altos porcentajes de utilización de carnes procesadas: oreada, ahumada y en embutidos, favorecida seguramente por factores culturales y posibilidad de compra en los expendios fronterizos.

Gráfico 1. Consumo de carne utilizada con más frecuencia

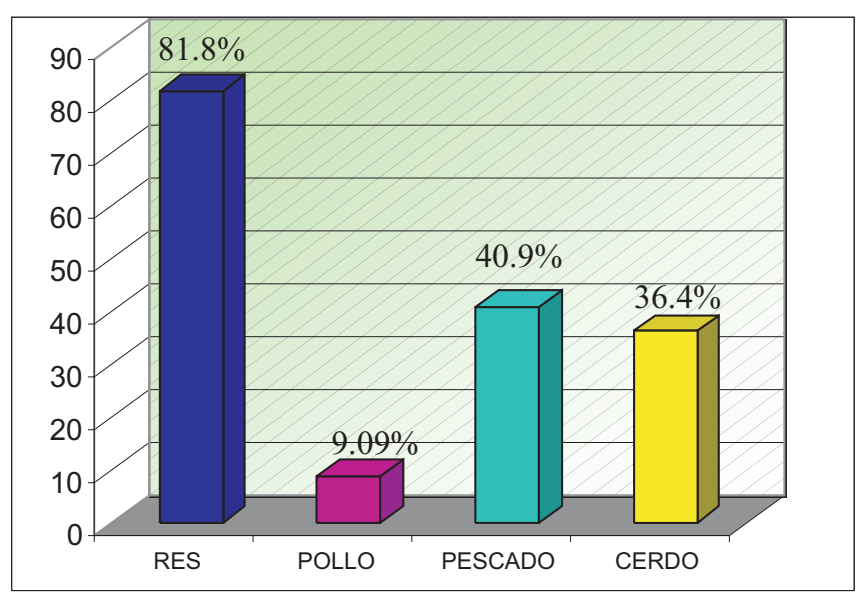

Respecto del consumo de fibra se encontró un importante porcentaje de consumo de frutas en $86 \%$, la fibra en $68 \%$ y vegetales en $77 \%$ en la población de estudio. Estos resultados son contrarios a lo que se esperaría en este grupo de pacientes, teniendo en cuenta que estudios anteriores han identificado estos alimentos como factores protectores para la prevención del cáncer de colon, como el realizado por Potter JD quien asevera que las verduras y frutas contienen un organizador de agentes potenciales de anticancerígenos, incluso los carotenos, las vitaminas $\mathrm{C}$ y $\mathrm{E}$ y el selenio, entre otras[7]. Igualmente, refieren el antecedente de utilización de productos naturales: ajo, y cebolla para sazonar las comidas 6.4 de cada 10 pacientes, frente a una tasa similar de utilización de condimentos artificiales. El ajo y la cebolla han sido identificados como agentes antimutagénicos o anticancerígenos ya que pueden inducir enzimas de desintoxicación que inhiben la formación del nitrosamina, manteniendo la formación de agentes antineoplásicos, diluyen y combinan carcinógenos en el tracto digestivo, alteran metabolismo de la hormona, y actúan como antioxidantes[4].

La adquisición de alimentos en ventas callejeras es referida por un bajo porcentaje de la población (36\%). Sin embargo se aprecia en $77 \%$ la práctica de consumo de alimentos recalentados, al igual que el consumo de arepas elaboradas con harina refinada de maíz, hábitos ampliamente difundidos en los hogares en el medio y considerados de alto riesgo para el evento en estudio.

Tabla 2. Hábitos intestinales presentes en la población de estudio

\begin{tabular}{|c|c|c|c|c|}
\hline \multirow{2}{*}{} & \multicolumn{2}{|c|}{ ESTRENIIMIENTO } & \multicolumn{2}{c|}{ DIARREA } \\
\cline { 2 - 5 } & $\mathrm{N}$ & $\%$ & $\mathrm{~N}$ & $\%$ \\
\hline $\mathrm{SI}$ & 19 & 86.4 & 17 & 77 \\
\hline $\mathrm{NO}$ & 3 & 13.6 & 5 & 23 \\
\hline TOTAL & 22 & 100 & 22 & 100 \\
\hline
\end{tabular}

Fuente: Entrevista

En relación con la información obtenida se determina que del total de los pacientes del estudio el 73\% presentaron estreñimiento y $32 \%$ refiere episodios de diarrea como síntomas precursores a la patología. Es de resaltar que al indagar sobre el uso de 
Comportamiento epidemiológico del cáncer de colon en el Área Metropolitana de Cúcuta 2001-2002

Alix Z Bohórquez O., Esperanza Gómez J., María Mercedes Ramírez O., Gloria Esperanza Zambrano P., Manuel Eduardo Moros

medicamentos que ayudan a controlar los síntomas, se encontró que $50 \%$ se automedica, empleando tanto medicamentos farmacológicos como el uso de la medicina casera, siendo los mas frecuentes el uso de sal de frutas, laxantes, hierbas(agua de senn) para el estreñimiento y terramicina y lomotil para los períodos de diarrea. Estas prácticas retardan la consulta médica y por la tanto el diagnóstico precoz y el tratamiento oportuno. Ver tablas 2 y 3.

Tabla 3. Antecedente y tipo de automedicación

\begin{tabular}{|l|c|c|}
\hline \multirow{2}{*}{ ANTECEDENTE } & \multicolumn{2}{|c|}{ AUTOMEDICACIÓN } \\
\cline { 2 - 3 } & $\mathrm{N}$ & $\%$ \\
\hline SI & 11 & 50 \\
\hline NO & 11 & 50 \\
\hline TOTAL & 22 & 100 \\
\hline \multicolumn{2}{|c|}{} \\
\hline TIPO & $\mathrm{N}$ & $\%$ \\
\hline SAL DE FRUTA & 3 & 27.3 \\
\hline HIERBAS & 4 & 36.4 \\
\hline LAXANTES & 3 & 27.3 \\
\hline ASA & 2 & 18.8 \\
\hline TERRAMICINA & 1 & 9.1 \\
\hline LOMOTIL & 1 & 9.1 \\
\hline ANALGÉSICOS & 1 & 9.1 \\
\hline
\end{tabular}

Fuente: Entrevista

Existen ciertos factores que dependen del estilo de vida y que predisponen a la aparición del cáncer de colon[4], por ello dentro de las conductas de riesgo presentes en los pacientes, se establece que más del $50 \%$ de los casos refieren sedentarismo y consumo de tabaco, y en menor proporción obesidad y alcoholismo. Al comparar los datos obtenidos respecto del tabaquismo en el presente estudio frente al realizado por Giovannucci E. y colaboradores se encontró en forma similar relación entre la presencia de cáncer de colon y el hábito de fumar, principal cancerígeno referido en la literatura[8].
Tabla 4. Factores de riesgo en el estilo de vida de los pacientes con cáncer de colon

\begin{tabular}{|r|c|c|c|c|c|c|c|c|}
\hline \multirow{2}{*}{} & \multicolumn{2}{|c|}{ OBESIDAD } & \multicolumn{2}{|c|}{ SEDENTARISMO } & \multicolumn{2}{|c|}{ ALCOHOLISMO } & \multicolumn{2}{|c|}{ TABAQUISMO } \\
\cline { 2 - 9 } & $\mathrm{N}$ & $\%$ & $\mathrm{~N}$ & $\%$ & $\mathrm{~N}$ & $\%$ & $\mathrm{~N}$ & $\%$ \\
\hline $\mathrm{SI}$ & 8 & 36.4 & 12 & 54.5 & 7 & 32 & 11 & 50 \\
\hline $\mathrm{NO}$ & 14 & 63.6 & 10 & 45.5 & 15 & 68 & 11 & 50 \\
\hline TOTAL & 22 & 100 & 10 & 100 & 22 & 100 & 22 & 100 \\
\hline
\end{tabular}

Fuente: Datos obtenidos de entrevista

Por otra parte $54.5 \%$ manifestaron ser sedentarios, confirmándose lo encontrado por Garabrant y colaboradores. Este es un factor determinante teniendo en cuenta que el sedentarismo aumenta el riesgo de cáncer al disminuir el peristaltismo intestinal, incrementándose el tiempo de exposición a los agentes cancerígenos[9]. Ver tabla 4.

Resaltando la información recolectada se encontró que dentro de las condiciones precursoras referidas por los pacientes se encuentran el sangrado rectal con $73 \%$, la pérdida de peso con $63.6 \%$, seguido por la hemorroides y la anemia con $36.4 \%$. La colitis ulcerativa, enfermedad inflamatoria del colon de causa desconocida y de naturaleza crónica, acompañada de tendencia o susceptibilidad a desarrollar cáncer de colon fue referida en menor proporción por la población de estudio, (27\%).

Respecto del tiempo de aparición de los síntomas relacionados con el cáncer de colon se encuentra que variaron desde 7 años y 1 mes hasta un caso que reporta evolución de los síntomas en quince días. De los 22 casos se establece que $91 \%$ consultó al médico, y de ellos $70 \%$ acudió con una frecuencia de más de 6 veces antes de que fuera diagnosticado el tumor.

Al analizar la realización de exámenes en la pesquisa diagnóstica de los casos se encuentra que los pacientes han sido valorados en mayor proporción a través de endoscopia digestiva alta, con $77.2 \%$, la biopsia con $45.4 \%$ y en un tercer lugar se encuentran la radiografía con $22.7 \%$, seguido por otros medios diagnósticos como Tomografía Axial Computarizada (TAC), Ecografía, Rectosigmoidoscopia, Colonoscopia, Colon 
Comportamiento epidemiológico del cáncer de colon en el Área Metropolitana de Cúcuta 2001-2002

Alix Z Bohórquez O., Esperanza Gómez J., María Mercedes Ramírez O.,, Gloria Esperanza Zambrano P., Manuel Eduardo Moros

por enema y la cirugía exploratoria. A pesar del largo periodo sintomatológico no se aprecia una valoración integral del paciente seguramente relacionada con la inaccesibilidad económica de los mismos o al desconocimiento de protocolos estandarizados para el diagnóstico de los casos.

Al analizar la localización del tumor se encontró que $27 \%$ se ubican en colon ascendente, $22 \%$ en forma similar en sigmoide y recto. Knaver y Silverman, 1888 refiere que los tumores se presentan en diferentes áreas de esa estructura, en las siguientes proporciones aproximadas: $16 \%$ en ciego y colon ascendente; $8 \%$ en colon transverso; 20 a $30 \%$ en colon descendente y sigmoide y 40 a $50 \%$ en el recto. Los adenocarcinomas, referidos en la literatura como los de mayor frecuencia, se confirmaron en $54 \%$ de los casos del estudio[10].

Respecto del tratamiento instaurado se encuentra que $43.2 \%$ de los casos recibió tratamiento médico o quirúrgico paliativo, el $37.8 \%$ de los casos fue sometido a cirugía con intenciones curativas, según la severidad del tumor y el estado general del paciente.

Gráfico 2. Tratamiento establecido a la población de estudio

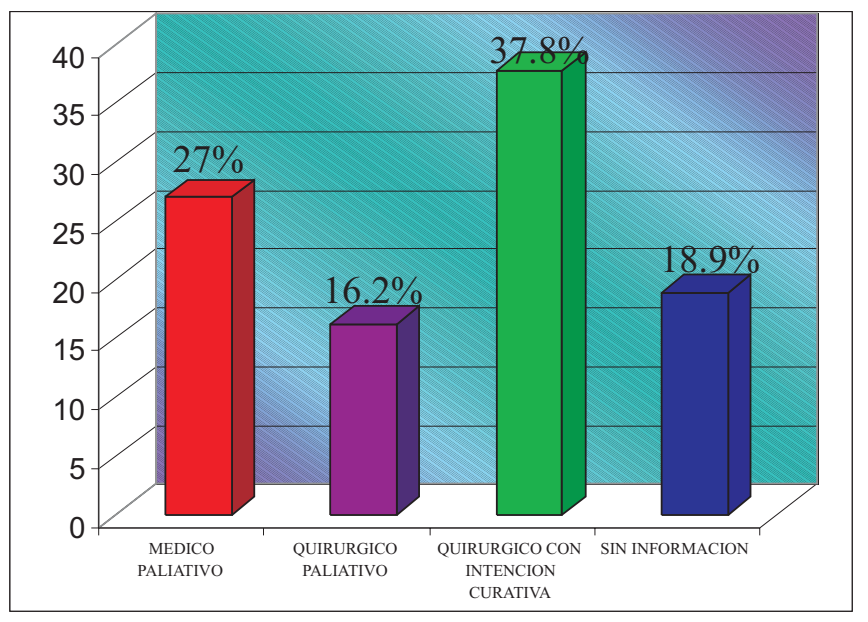

Fuente: Datos obtenidos de historias clínicas

Al analizar el antecedente de terapia neoadyuvante (antes de la cirugía) se encontró que cinco (5) pacientes fueron sometidos a quimioterapia y 4 a radioterapia, no encontrándose similitud entre el número de ciclos o en las dosis de radioterapia administradas.

De otra parte la terapia adyuvante (después de la cirugía) fue administrada así: 10 pacientes sometidos a quimioterapia (no existe información completa sobre número y cumplimiento de los ciclos), y 7 pacientes sometidos a radioterapia entre 15 y 36 sesiones. Los pacientes reportaron que el dolor y la falta de tiempo para sacar citas y cumplirlas contribuyeron a que no se realizaran los esquemas completos de las mismas.

Al analizar los tratamientos quirúrgicos se reportaron con frecuencias que varían entre 1 y 2 los siguientes procedimientos: sigmoidectomía, hemicolectomía derecha, colectomía derecha, hemicolectomía izquierda, colostomía. Este último se encontró positivo en 9 pacientes del estudio.

\section{CONCLUSIONES}

Respecto de las características epidemiológicas del cáncer de colon se concluye en el presente estudio que los casos se incrementan con la edad observándose el mayor número de casos a partir de los 50 años, con un porcentaje similar en ambos sexos y baja escolaridad. Se reportó el antecedente familiar de cáncer de colon en 0.8 de cada 10 pacientes del estudio. Respecto de la evolución de la enfermedad un alto porcentaje manifiesta haber tenido estreñimiento, diarrea o ambos síntomas. El 50\% refiere automedicación para el manejo de estos síntomas, empleando tanto medicamentos farmacológicos, como el uso de la medicina casera. Como condiciones precursoras se encuentran el sangrado rectal con un $73 \%$, la pérdida de peso con $63.6 \%$, seguido por la hemorroides y la anemia con $36.4 \%$.

Respecto del tumory su manejo se encontraron largos períodos de evolución de la enfermedad y alta frecuencia de asistencia a las consultas médicas antes de que se definiera el diagnóstico. La pesquisa 
Comportamiento epidemiológico del cáncer de colon en el Área Metropolitana de Cúcuta 2001-2002

Alix Z Bohórquez O., Esperanza Gómez J., María Mercedes Ramírez O., Gloria Esperanza Zambrano P., Manuel Eduardo Moros

diagnóstica se realiza especialmente a través de la endoscopia digestiva alta y otros medios de diagnóstico, sin observarse exhaustividad ni cumplimiento de protocolos de diagnóstico en la mayoría de los casos. En concordancia con lo anterior, el tratamiento instaurado fue paliativo médico o quirúrgico en un $43.2 \%$ de los casos y el $37.8 \%$ de los casos fue sometido a cirugía con intenciones curativas. El $54 \%$ de los tumores fueron clasificados como adenocarcinomas. Se encuentra una baja frecuencia de aplicación de quimio y radio terapia neoadyuvante, incrementándose estos tratamientos después de la cirugía. Los pacientes reportaron que el dolor y la falta de tiempo para sacar citas y cumplirlas contribuyeron a que no se realizaran los esquemas completos de las mismas.

Se identificaron como factores de riesgo el alto consumo de carne de res en la dieta diaria, con importante ingesta de alimentos embutidos, la reutilización de los aceites en la cocción de los alimentos, el consumo de comidas recalentadas. Es ampliamente utilizada en la dieta la harina refinada de maíz para la elaboración y consumo de arepas. Los factores de riesgo en el estilo de vida que se presentaron con mayor porcentaje fueron el sedentarismo y el tabaquismo, seguido del consumo de alcohol y la obesidad.

\section{BIBLIOGRAFIA}

1. BOND John H. Prevention, Screening, Treatment, and Surveillance for High-Risk. COLORECTAL CANCER UPDATE Medical Clinics of North America Advances in Gastroenterology. Volume 84, Number 5, September 2000.

2. Estadísticas Servicio de Salud de Norte de Santander.

3. AGA Technical Revew on Hereditary Colorectal Cáncer and Genetic Tesfing. Gastroenterology 2001 ; $121 ; 198-213$.
4. SANDLER, Robert S. MD. COLORECTAL NEOPLASIA, PART 1: THE SCIENTIFIC BASIS FOR CURRENT MANAGEMENT. Epidemiology and risk 4 Factors for colorectal cancer. Gastroenterology clinics. Volumen 25. Number 4. December 1996.

5. YOUNG-IN KIM 1 M.D., FRCP(C) AGA Technical Review: Impact of Dietary Fiber on Colon Cancer Occurrence. Gastroenterology. Volume 118, Number 6, June 1, 2000.

6. GIOVANNUCCI E., RIMM EB, STAMPFER MJ et al Intake of fat, meat and fiber in relation to risk of colon cancer in men. Cancer Res 54:2390, 1994.

7. POTTER JD: Dietary fiber, vegetables et cancer. J Nutr 118: 1591, 1988.

8. GIOVANNUCCI E., et al. A Prospective study of cigarette smoking and risk of colorectal adenoma and colorectal cancer in U.s. woman J.Natl cancer inst 86:183, 1994.

9. GARABRAND DH. et al. Job activity and colon cancer risk. Am J Epidemiol 1 19:1005, 1984.

10. WASSERMAN, Ernesto. Detección precoz de cáncer de colon. Cvitko vic \& associates consultants. Argentina. Instituto Alexander Flemming. 